\title{
FREE BOUNDARY REGULARITY NEAR THE FIXED BOUNDARY FOR THE FULLY NONLINEAR OBSTACLE PROBLEM
}

\author{
EMANUEL INDREI
}

\begin{abstract}
The interior free boundary theory for linear elliptic operators in higher dimensions was developed by Caffarelli [Caf77] in the low regularity context. In these notes, the up-to-the boundary free boundary regularity is discussed for nonlinear elliptic operators based on a different approach.
\end{abstract}

\section{Introduction}

Caffarelli proved that if $L$ is a linear uniformly elliptic operator and $u \geq 0$ solves

$$
L\left(D^{2} u\right)=\chi_{\{u>0\}} \quad \text { in } B_{1}
$$

then for $x \in \Gamma=\partial\{u>0\} \cap B_{1}$ with positive Lebesgue density for $\{u=0\}$, i.e. satisfying

$$
\liminf _{r \rightarrow 0^{+}} \frac{\left|B_{r}(x) \cap\{u=0\}\right|}{\left|B_{r}(x)\right|}>0 \quad 1
$$

there is a Lipschitz function $g$ such that $\Gamma \cap B_{s}(x)$ admits a representation with respect to $g$ in a coordinate system for some $s>0$. The Lipschitz regularity can be improved to $C^{1}$ and higher regularity follows (up to analyticity) via a theorem of Kinderlehrer and Nirenberg [KN77]. Caffarelli's theorem is optimal in the sense that there exists a solution when $L=\Delta$ for which there is a free boundary point with zero Lebesgue density for $\{u=0\}$ and in a neighborhood

$\left.\overline{{ }^{1} B_{r}(x) \cap\{u}=0\right\} \mid \geq a r^{n}$ for $r>0$ small enough is usually assumed for convenience; $\limsup _{r \rightarrow 0^{+}} \frac{\left|B_{r}(x) \cap\{u=\overline{0}\}\right|}{\left|B_{r}(x)\right|}>0$ is sufficient. 
of the point the free boundary develops a cusp singularity and is not a graph in any system of coordinates [Sch77].

In a recent work [Ind19], the author proved that for solutions of (1.1) with zero Dirichlet boundary data, with $L$ replaced by a convex fully nonlinear uniformly elliptic operator $F$, if $x \in \partial B_{1} \cap \bar{\Gamma}$, then $\Gamma$ can be represented as the graph of a $C^{1}$ function in a neighborhood of $x$. There are two surprising differences between the interior and boundary result: first, there are no density assumptions in the boundary case (in particular, cusp-type singularities do not exist); second, there is an example which generates a free boundary which is $C^{1}$ with a specific Dini modulus of continuity for the free normal (see e.g. [PSU12, Remark 8.8]).

In his original approach, Caffarelli estimated pure second derivatives from below. The linear approach developed thereafter to handle regularity near the fixed boundary involves specific barrier constructions involving the operator and monotonicity formulas [Ura96, SU03, And07]. The nonlinear method is based on understanding a maximal mixed partial derivative along a preferred direction.

In what follows, $F$ satisfies

- $F(0)=0$.

- $F$ is uniformly elliptic with ellipticity constants $\lambda_{0}, \lambda_{1}>0$ such that

$$
\mathcal{P}^{-}(M-N) \leq F(M)-F(N) \leq \mathcal{P}^{+}(M-N)
$$

where $M$ and $N$ are symmetric matrices and $\mathcal{P}^{ \pm}$are the Pucci operators

$$
\mathcal{P}^{-}(M):=\inf _{\lambda_{0} \leq N \leq \lambda_{1}} \operatorname{tr}(N M), \quad \mathcal{P}^{+}(M):=\sup _{\lambda_{0} \leq N \leq \lambda_{1}} \operatorname{tr}(N M)
$$

- $F$ is convex and $C^{1}$.

Let $\Omega$ be an open set and $B_{r}^{+}=\left\{x:|x|<r, x_{n}>0\right\}$. A continuous function $u$ belongs to $P_{r}^{+}(0, M, \Omega)$ if $u$ satisfies in the viscosity sense

- $F\left(D^{2} u\right)=\chi_{\Omega}$ in $B_{r}^{+}$ 
- $\|u\|_{L^{\infty}\left(B_{r}^{+}\right)} \leq M$

- $u=0$ on $\left\{x_{n}=0\right\} \cap \overline{B_{1}^{+}}=: B_{1}^{\prime}$.

In [IM16a] it was shown that $W^{2, p}$ solutions are $C^{1,1}$ (see also [FS14, IM16b] for the interior case). Furthermore, given $u \in P_{r}^{+}(0, M, \Omega)$, the free boundary is denoted by $\Gamma=\partial \Omega \cap B_{r}^{+}$.

A blow-up limit of $\left\{u_{j}\right\} \subset P_{1}^{+}(0, M, \Omega)$ is a limit of the form

$$
\lim _{k \rightarrow \infty} \frac{u_{j_{k}}\left(s_{k} x\right)}{s_{k}^{2}}
$$

where $\left\{j_{k}\right\}$ is a subsequence of $\{j\}$ and $s_{k} \rightarrow 0^{+}$.

In $\$ 2$, non-transversal intersection is shown for $\Omega=(\{u \neq 0\} \cup\{\nabla u \neq 0\}) \cap$ $\left\{x_{n}>0\right\}$ and a problem in superconductivity is discussed in which $\Omega=\{\nabla u \neq$ $0\}) \cap\left\{x_{n}>0\right\}$; in $\left\{3, C^{1}\right.$ regularity is proved when $u \geq 0$; last, some of the technical details are shown in the appendix $\$ 4$.

\section{Non-transversal intersection and Classification OF BLOW-UP} LIMITS

One of the main results discussed in this section is the following.

Theorem 2.1. There exists $r_{0}>0$ and a modulus of continuity $\omega$ such that

$$
\Gamma(u) \cap B_{r_{0}}^{+} \subset\left\{x: x_{n} \leq \omega\left(\left|x^{\prime}\right|\right)\left|x^{\prime}\right|\right\}
$$

for all $u \in P_{1}^{+}(0, M, \Omega)$ provided $0 \in \overline{\Gamma(u)}$ and $\Omega=(\{u \neq 0\} \cup\{\nabla u \neq 0\}) \cap\left\{x_{n}>\right.$ $0\}$.

If one varies the boundary data, then non-transversal intersection may not hold [And07, see Examples 3 \& 4]. The difficulty in the fully nonlinear context is that monotonicity formulas are not available and a classification of blow-up limits requires a new approach: if blow-up limits are not half-space solutions, then a certain regularity property holds. More precisely: 
Proposition 2.2. Suppose $\Omega=(\{u \neq 0\} \cup\{\nabla u \neq 0\}) \cap\left\{x_{n}>0\right\}$ and $\left\{u_{j}\right\} \subset$ $P_{1}^{+}(0, M, \Omega)$. If $0 \in \overline{\left\{u_{j} \neq 0\right\}}$ and $\nabla u_{j}(0)=0$, then one of the following is true: (i) all blow-up limits of $\left\{u_{j}\right\}$ at the origin are of the form $u_{0}(x)=b x_{n}^{2}$ for $b>0$; (ii) there exists $\left\{u_{k_{j}}\right\} \subset\left\{u_{j}\right\}$ such that for all $R \geq 1$, there exists $j_{R} \in \mathbb{N}$ such that for all $j \geq j_{R}$,

$$
u_{k_{j}} \in C^{2, \alpha}\left(B_{\frac{R r_{j}}{4}}^{+}\right),
$$

where the sequence $\left\{r_{j}\right\}$ depends on $\left\{u_{j}\right\}$.

The proof relies on the fact that if not all blow-up solutions are half-space solutions, then one can construct a specific sequence producing a limit of the form $a x_{1} x_{n}+b x_{n}^{2}$.

Proposition 2.3. Let $\left\{u_{j}\right\} \subset P_{1}^{+}(0, M, \Omega)$ and suppose $0 \in \overline{\left\{u_{j} \neq 0\right\}},\left\{\nabla u_{j} \neq\right.$ $0\} \cap\left\{x_{n}>0\right\} \subset \Omega, \nabla u_{j}(0)=0$. Then one of the following is true:

(i) all blow-up limits of $\left\{u_{j}\right\}$ at the origin are of the form $u_{0}(x)=b x_{n}^{2}$ for some $b>0$

(ii) there exists a blow-up limit of $\left\{u_{j}\right\}$ of the form $a x_{1} x_{n}+b x_{n}^{2}$ for $a \neq 0, b \in \mathbb{R}$.

Proof. Let

$$
N:=\limsup _{|x| \rightarrow 0, x_{n}>0} \frac{1}{x_{n}} \sup _{u \in\left\{u_{j}\right\}} \sup _{e \in \mathbb{S}^{n-2} \cap e_{n}^{\perp}} \partial_{e} u(x)
$$

and consider a sequence $\left\{x^{k}\right\}_{k \in \mathbb{N}}$ with $x_{n}^{k}>0, u_{j_{k}} \in\left\{u_{j}\right\}$, and $e^{k} \in \mathbb{S}^{n-2} \cap e_{n}^{\perp}$ such that the previous limit is given by

$$
\lim _{k \rightarrow \infty} \frac{1}{x_{n}^{k}} \partial_{e^{k}} u_{j_{k}}\left(x^{k}\right) .
$$

Note that $N<\infty$ by $C^{1,1}$ regularity for the class $P_{1}^{+}(0, M, \Omega)$ and the boundary condition (see [IM16a]). By compactness, $e^{k} \rightarrow e_{1} \in \mathbb{S}^{n-2}$ (along a subsequence) so that up to a rotation,

$$
N=\lim _{k \rightarrow \infty} \frac{1}{x_{n}^{k}} \partial_{x_{1}} u_{j_{k}}\left(x^{k}\right) .
$$


Next, if

$$
\tilde{u}_{j}(x):=\frac{u_{k_{j}}\left(s_{j} x\right)}{s_{j}^{2}} \rightarrow u_{0}(x)
$$

for some sequence $s_{j} \rightarrow 0^{+}$, where the convergence is in $C_{l o c}^{1, \alpha}\left(\mathbb{R}_{+}^{n}\right)$ for any $\alpha \in$ $[0,1), u_{0} \in C^{1,1}\left(\mathbb{R}_{+}^{n}\right)$ satisfies the following PDE in the viscosity sense

$$
\begin{cases}F\left(D^{2} u_{0}\right)=1 & \text { a.e. in } \mathbb{R}_{+}^{n} \cap \Omega_{0} \\ \left|\nabla u_{0}\right|=0 & \text { in } \mathbb{R}_{+}^{n} \backslash \Omega_{0} \\ u=0 & \text { on } \mathbb{R}_{+}^{n-1},\end{cases}
$$

where $\Omega_{0}=\left\{\nabla u_{0} \neq 0\right\} \cap\left\{x_{n}>0\right\}$. Note that

$$
N \geq \lim _{j}\left|\frac{\partial_{x_{i}} u_{k_{j}}\left(s_{j} x\right)}{s_{j} x_{n}}\right|=\lim _{j}\left|\frac{\partial_{x_{i}} \tilde{u}_{j}(x)}{x_{n}}\right|=\left|\frac{\partial_{x_{i}} u_{0}(x)}{x_{n}}\right|
$$

for all $i \in\{1, \ldots, n-1\}$. If $N=0$, then $\partial_{x_{i}} u_{0}=0$ for all $i \in\{1, \ldots, n-1\}$ so that $u_{0}(x)=u_{0}\left(x_{n}\right)$ and the conditions readily imply $u_{0}\left(x_{n}\right)=b x_{n}^{2}$. Since $N$ does not depend on the sequence $\left\{s_{j}\right\}$ it follows that in this case all blow-up limits have the previously stated form. Suppose that $N>0$, let $r_{k}=\left|x^{k}\right|$, and consider the re-scaling of $u_{j_{k}}$ with respect to $r_{k}$. Note that along a subsequence, $y^{k}:=\frac{x^{k}}{r_{k}} \rightarrow y \in \mathbb{S}^{n-1}$. By the choice of $r_{k}$,

$$
\lim _{k \rightarrow \infty} \frac{v\left(y^{k}\right)}{y_{n}^{k}}=\lim _{k \rightarrow \infty} \frac{\partial_{x_{1}} \tilde{u}_{k}\left(y^{k}\right)}{y_{n}^{k}}=\lim _{k \rightarrow \infty} \frac{\partial_{x_{1}} u_{j_{k}}\left(r_{k} y^{k}\right)}{r_{k} y_{n}^{k}}=N,
$$

where $v=\partial_{x_{1}} u_{0}$. In particular,

$$
v(y)=N y_{n}
$$

and by an argument in [IM16a] (involving the boundary Harnack inequality), $u_{0}(x)=a x_{1} x_{n}+b x_{n}^{2}$ with $a \neq 0$.

Proof of Proposition 2.2. Either all blow-up limits are of the form $u_{0}(x)=b x_{n}^{2}$ or there exists a subsequence

$$
\tilde{u}_{j}(x)=\frac{u_{k_{j}}\left(r_{j} x\right)}{r_{j}^{2}}
$$


producing a limit of the form $u_{0}(x)=a x_{1} x_{n}+b x_{n}^{2}$ for $a>0$ (up to a rotation). Let $c=c(a, b)$ be the constant from Lemma 4.4 and note that since $\tilde{u}_{j} \rightarrow u_{0}$ in $C_{l o c}^{1, \alpha}$, there exists $j_{0}=j_{0}(a, R) \in \mathbb{N}$ such that for every cylinder $S_{(\alpha, \beta)}\left(e_{1}\right)$ there exists $x \in S_{(\alpha, \beta)}\left(e_{1}\right) \cap B_{R}^{+}$such that $\left|\nabla \tilde{u}_{j}(x)\right| \geq \frac{c}{2}$ for all $j \geq j_{0}$, where $R \geq 1$. Choose a constant $C_{0}=C_{0}(a, b, R)>0$ such that

$$
C_{0} \partial_{x_{1}} u_{0}-u_{0} \geq 0
$$

in $B_{R}^{+}$and $j_{0}^{\prime} \geq j_{0}$ for which

$$
C_{0} \partial_{x_{1}} \tilde{u}_{j}-\tilde{u}_{j} \geq 0 \quad \text { in } B_{\frac{R}{2}}^{+}
$$

whenever $j \geq j_{0}^{\prime}$ by Lemma 4.1. Now fix $j \geq j_{0}^{\prime}$ and suppose $z \in \Gamma_{i}\left(\tilde{u}_{j}\right) \cap B_{\frac{R}{2}}^{+}$. Then there exists a ball $B \subset \operatorname{int}\left\{\tilde{u}_{j}=0\right\} \cap B_{\frac{R}{2}}^{+}$and a cylinder $S$ in the $e_{1^{-}}$ direction generated by $B$. Now select $x \in S \cap B_{R}^{+}$for which $\left|\nabla \tilde{u}_{j}(x)\right|>0$ and $-R<x_{1}<-R / 2$. In particular, there exists a small ball around $x$, say $\tilde{B}$ such that $F\left(D^{2} \tilde{u}_{j}\right)=1$ in $\tilde{B}$ and one may assume $\tilde{B} \subset\left\{\tilde{u}_{j} \neq 0\right\}$. Note that $\tilde{B}$ is contained in the cylinder $S$ and let $E_{t}=\tilde{B}+t e_{1}$ for $t \in \mathbb{R}$. If $t>0$ is such that $\overline{E_{t}} \cap\left\{\tilde{u}_{j}=0\right\} \neq \emptyset$, and for all $0 \leq s<t, E_{s} \cap\left\{\tilde{u}_{j}=0\right\}=\emptyset$, choose $y \in \overline{E_{t}} \cap\left\{\tilde{u}_{j}=0\right\}$. If $\tilde{u}_{j}>0$ in $\tilde{B}$, then by 2.3 it follows that $\tilde{u}_{j}$ is strictly positive at a point in $\left\{\tilde{u}_{j}=0\right\}$, a contradiction. Thus $\tilde{u}_{j}<0$ in $\tilde{B}$. By convexity of $F$

$$
a_{k l} \partial_{k l} \tilde{u}_{j} \geq 0 \text { in } E_{t}
$$

Since $0=\tilde{u}_{j}(y)>\tilde{u}_{j}(x)$ for $x \in E_{t}$ and $y$ satisfies an interior ball condition, then Hopf's lemma implies that $\frac{\partial}{\partial n} \tilde{u}_{j}(y)>0$, where $n$ is the outer normal to the ball at $y$. If there exists $z \in B_{\delta}(y)$ such that $\tilde{u}_{j}(z)>0$, then this contradicts the monotonicity, if $\delta>0$ is sufficiently small: $\overline{E_{\eta}} \subset B \subset \operatorname{int}\left\{\tilde{u}_{j}=0\right\}$ for $\eta>0$ large enough and since $\tilde{u}_{j}(z)>0$, the monotonicity (2.3) implies that $\tilde{u}_{j}\left(z+e_{1} s\right)>0$, for some $s>0$ such that $z+e_{1} s \in\left\{\tilde{u}_{j}=0\right\}$. Hence, $\tilde{u}_{j} \leq 0$ on $B_{\delta}(y)$ and thus 
$\nabla \tilde{u}_{j}(y)=0$, a contradiction. The conclusion is that for $j \geq j_{0}^{\prime}$,

$$
\Gamma_{i}\left(\tilde{u}_{j}\right) \cap B_{\frac{R}{2}}^{+}=\emptyset .
$$

In particular, $\left(B_{\frac{R}{2}}^{+} \backslash \Omega_{j}\right)^{o}=\emptyset$ and non-degeneracy implies that $\left|B_{\frac{R}{2}}^{+} \backslash \Omega_{j}\right|=0$. Thus the $C^{1,1}$ function $\tilde{u}_{j}$ satisfies $F\left(D^{2} \tilde{u}_{j}\right)=1$ in $B_{\frac{R}{2}}^{+}$in the viscosity sense and the up to the boundary Evans-Krylov theorem (see e.g. [Saf94]) implies that $\tilde{u}_{j} \in C^{2, \alpha}\left(B_{\frac{R}{4}}^{+}\right)$. In particular, $u_{k_{j}} \in C^{2, \alpha}\left(B_{\frac{R r_{j}}{4}}^{+}\right)$.

Theorem 2.4. Suppose $u \in P_{1}^{+}(0, M, \Omega)$ and $\Omega=(\{u \neq 0\} \cup\{\nabla u \neq 0\}) \cap\left\{x_{n}>\right.$ $0\}$. If $0 \in \overline{\{u \neq 0\}}$ and $\nabla u(0)=0$, then the blow-up limit of $u$ at the origin has the form

$$
u_{0}(x)=a x_{1} x_{n}+b x_{n}^{2}
$$

for $a, b \in \mathbb{R}$.

Proof. By Proposition 2.2, either $u_{0}(x)=b x_{n}^{2}$ or $D^{2} u(0)$ exists and the rescaling of $u$ is given by

$$
u_{j}(x)=\frac{u\left(r_{j} x\right)}{r_{j}^{2}}=\left\langle x, D^{2} u(0) x\right\rangle+o(1) .
$$

Since $u_{0}\left(x^{\prime}, 0\right)=0$ for $x^{\prime} \in \mathbb{R}^{n-1}$, it follows that $u_{0}$ has the claimed form (up to a rotation).

Theorem 2.5. Suppose $\Omega=(\{u \neq 0\} \cup\{\nabla u \neq 0\}) \cap\left\{x_{n}>0\right\}, 0 \in \bar{\Gamma}$, and $\left\{u_{j}\right\} \subset P_{1}^{+}(0, M, \Omega)$. Then the blow-up limit of $\left\{u_{j}\right\}$ at the origin has the form

$$
u_{0}(x)=b x_{n}^{2}
$$

for $b>0$.

Proof. By Proposition 2.2, either $u_{0}(x)=b x_{n}^{2}$ or there exists a subsequence

$$
u_{k_{j}}(x) \in C^{2, \alpha}\left(B_{\frac{R r_{j}}{4}}^{+}\right)
$$


which contradicts that $F$ is continuous (consider a sequence of points approaching the free boundary from the set where the equation is satisfied with the right-handside being equal to one and from the complement).

Remark 2.1. There exist global solutions which are not blow-up solutions (at contact points).

proof of Theorem 2.1. It suffices to show that for any $\epsilon>0$ there exists $\rho_{\epsilon}>0$ such that $\Gamma(u) \cap B_{\rho_{\epsilon}}^{+} \subset B_{\rho_{\epsilon}}^{+} \backslash \mathcal{C}_{\epsilon}$, where $\mathcal{C}_{\epsilon}=\left\{x_{n}>\epsilon\left|x^{\prime}\right|\right\}$. If not, then there exists $\epsilon>0$ such that for all $k \in \mathbb{N}$ there exists $u_{k} \in P_{1}^{+}(0, M, \Omega)$ with

$$
\Gamma\left(u_{k}\right) \cap B_{1 / k}^{+} \cap \mathcal{C}_{\epsilon} \neq \emptyset
$$

where $0 \in \overline{\Gamma\left(u_{k}\right)}$. If all blow-ups of $\left\{u_{k}\right\}$ are half-space solutions. Let $x_{k} \in$ $\Gamma\left(u_{k}\right) \cap B_{1 / k}^{+} \cap \mathcal{C}_{\epsilon}$ and set $y_{k}=\frac{x_{k}}{r_{k}}$ with $r_{k}=\left|x_{k}\right|$. Consider $\tilde{u}_{k}(x)=\frac{u_{k}\left(r_{k} x\right)}{r_{k}^{2}}$ so that $y_{k} \in \Gamma\left(\tilde{u}_{k}\right), \tilde{u}_{k} \rightarrow b x_{n}^{2}, y_{k} \rightarrow y \in \partial B_{1} \cap C_{\epsilon}$ (up to a subsequence), and $y \in \Gamma\left(u_{0}\right)$, a contradiction. Second, select a subsequence $\left\{u_{k_{j}}\right\}$ of $\left\{u_{k}\right\}$ such that for all $j \geq j_{2}$, $u_{k_{j}} \in C^{2, \alpha}\left(B_{\frac{r_{j}}{2}}^{+}\right)$, where $j_{2} \in \mathbb{N}$ and the sequence $\left\{r_{j}\right\}$ depends on $\left\{u_{k}\right\}$. Since $0 \in \overline{\Gamma\left(u_{k_{j}}\right)}$, there exists

$$
x_{j} \in \Gamma\left(u_{k_{j}}\right) \cap B_{\frac{r_{j}}{2}}^{+}
$$

which contradicts the continuity of $F$ (consider a sequence of points approaching the free boundary from the set where the equation is satisfied with the righthand-side being equal to one and from the complement).

\subsection{An obstacle problem in superconductivity. Equations of the type}

$$
F\left(D^{2} u, x\right)=g(x, u) \chi_{\{\nabla u \neq 0\}}
$$

have been investigated in [CS02 and are based on physical models, e.g. the stationary equation for the mean-field theory of superconducting vortices when the scalar stream is a function of the scalar magnetic potential Cha95, CRS96, ESS98. It is shown that in certain configurations in two dimensions, the set 
$\{\nabla u=0\}$ is convex. In a recent paper Ind18, the author proved non-transversal intersection for $\Omega=\{\nabla u \neq 0\} \cap\left\{x_{2}>0\right\}$. If $\{u<0\}$ has sufficiently small density, non-transversal intersection follows from the techniques discussed above without a dimension restriction: suppose

$$
\frac{\left|\{u<0\} \cap B_{r}^{+}\right|}{\left|B_{r}^{+}\right|} \rightarrow 0
$$

as $r \rightarrow 0^{+}$. A limit of the form

$$
u_{0}(x)=\lim _{k \rightarrow \infty} \frac{u_{j_{k}}\left(s_{k} x\right)}{s_{k}^{2}}
$$

satisfies $u_{0} \geq 0$ and therefore cannot be $a x_{1} x_{n}+b x_{n}^{2}$ for $a \neq 0$. In particular, it must be a half-space solution by Proposition 2.3 and the non-transversal intersection follows as before. The assumption on the negativity set appeared in [MM04] where the authors considered the non-transversal intersection subject to additional assumptions on the operator and solution.

\section{3. $C^{1}$ REGULARITY}

In the physical case when $u \geq 0$, the free boundary is $C^{1}$ without density assumptions.

Theorem 3.1. Let $u \in P_{1}^{+}(0, M, \Omega)$ be non-negative, $\Omega=(\{u \neq 0\} \cup\{\nabla u \neq$ $0\}) \cap\left\{x_{n}>0\right\}$, and $0 \in \overline{\Gamma(u)}$. There exists $r_{0}>0$ such that $\Gamma$ is the graph of $a$ $C^{1}$ function in $B_{r_{0}}^{+}$.

Proof. First, for any $\epsilon>0$ there exists $r(\epsilon, M)>0$ such that if $x^{0} \in \Gamma(u) \cap B_{1 / 2}^{+}$ and $d=x_{n}^{0}<r$, then

$$
\sup _{B_{2 d}^{+}\left(x^{0}\right)}|u-h| \leq \epsilon d^{2}, \quad \sup _{B_{2 d}^{+}\left(x^{0}\right)}|\nabla u-\nabla h| \leq \epsilon d
$$

where

$$
h(x)=b\left[\left(x_{n}-d\right)^{+}\right]^{2},
$$


and $b>0$ depends on the ellipticity constants of $F$. If not, then there exists $\epsilon>0$, non-negative $u_{j} \in P_{1}^{+}(0, M, \Omega)$, and $x^{j} \in \Gamma\left(u_{j}\right) \cap B_{1 / 2}^{+}$with $d_{j}=x_{n}^{j} \rightarrow 0$, for which

$$
\sup _{B_{2 d_{j}}\left(x^{j}\right)^{+}}\left|u_{j}-b\left[\left(x_{n}-d_{j}\right)^{+}\right]^{2}\right|>\epsilon d_{j}^{2}
$$

or

$$
\sup _{B_{2 d_{j}}\left(x^{j}\right)^{+}}\left|\nabla u_{j}-2 b\left(x_{n}-d_{j}\right)^{+}\right|>\epsilon d_{j} .
$$

Let $\tilde{u}_{j}(x)=\frac{u_{j}\left(\left(x^{j}\right)^{\prime}+d_{j} x\right)}{d_{j}^{2}}$ so that in particular

$$
\left\|\tilde{u}_{j}-h\right\|_{C^{1}\left(B_{2}^{+}\left(e_{n}\right)\right)} \geq \epsilon,
$$

where $h(x)=b\left[\left(x_{n}-1\right)^{+}\right]^{2}$. Since $\tilde{u}_{j}\left(e_{n}\right)=\left|\nabla \tilde{u}_{j}\left(e_{n}\right)\right|=0$, the $C^{1,1}$ regularity of $\tilde{u}_{j}$ implies that $\left|\tilde{u}_{j}(x)\right| \leq C\left|x-e_{n}\right|^{2}$. By passing to a subsequence, if necessary,

$$
\tilde{u}_{j} \rightarrow u_{0}
$$

where $u_{0} \in C^{1,1}\left(\mathbb{R}_{+}^{n}\right)$ satisfies the following PDE in the viscosity sense

$$
\begin{cases}F\left(D^{2} u_{0}\right)=1 & \text { a.e. in } \mathbb{R}_{+}^{n} \cap \Omega_{0}, \\ \left|\nabla u_{0}\right|=0=u_{0} & \text { in } \mathbb{R}_{+}^{n} \backslash \Omega_{0}, \\ u_{0}=0 & \text { on } \mathbb{R}_{+}^{n-1} .\end{cases}
$$

Now let

$$
N=\limsup _{|x| \rightarrow 0, x_{n}>0} \frac{1}{x_{n}} \sup _{u \in P_{1}^{+} \cap\{u \geq 0\}} \sup _{e \in \mathbb{S}^{n-2} \cap e_{\frac{1}{n}}^{-1}} \sup _{y \in B_{1 / 2}^{+} \cap\left\{x_{n}=0\right\}} \partial_{e} u(x+y)
$$

and note that $N<\infty$ by $C^{1,1}$ regularity and the boundary condition: for any $e \in \mathbb{S}^{n-2} \cap e_{n}^{\perp}$ and $y \in \overline{B_{1 / 2}^{+}} \cap\left\{x_{n}=0\right\}$, it follows that $\partial_{e} u\left(x^{\prime}+y\right)=0$. Furthermore,

$$
N \geq \lim _{j}\left|\frac{\partial_{x_{i}} u_{j}\left(d_{j} x+\left(x^{j}\right)^{\prime}\right)}{d_{j} x_{n}}\right|=\lim _{j}\left|\frac{\partial_{x_{i}} \tilde{u}_{j}(x)}{x_{n}}\right|=\left|\frac{\partial_{x_{i}} u_{0}(x)}{x_{n}}\right|
$$


for all $i \in\{1, \ldots, n-1\}$. In particular, let $v=\partial_{x_{1}} u_{0}$ so that in $\mathbb{R}_{+}^{n}$,

$$
|v(x)| \leq N x_{n}
$$

If $N=0$, then $\partial_{x_{i}} u_{0}=0$ for all $i \in\{1, \ldots, n-1\}$ and therefore $u_{0}(x)=u_{0}\left(x_{n}\right)$. Since $e_{n}$ is a free boundary point, it follows that $u_{0}=h$, a contradiction. Thus $N>0$ and there is a sequence $\left\{x^{k}\right\}_{k \in \mathbb{N}}$ with $x_{n}^{k}>0, u_{k} \in P_{1}^{+}(0, M, \Omega), u_{k} \geq 0$, $y^{k} \in \overline{B_{1 / 2}^{+}} \cap\left\{x_{n}=0\right\}$, and $e^{k} \in \mathbb{S}^{n-2} \cap e_{n}^{\perp}$ such that

$$
N=\lim _{k \rightarrow \infty} \frac{1}{x_{n}^{k}} \partial_{e^{k}} u_{k}\left(x^{k}+y^{k}\right) .
$$

By compactness, $e^{k} \rightarrow e_{1} \in \mathbb{S}^{n-2}$ (along a subsequence) so that up to a rotation,

$$
N=\lim _{k \rightarrow \infty} \frac{1}{x_{n}^{k}} \partial_{x_{1}} u_{k}\left(x^{k}+y^{k}\right) .
$$

Let

$$
\tilde{u}_{k}(x)=\frac{u_{k}\left(y^{k}+r_{k} x\right)}{r_{k}^{2}},
$$

where $r_{k}=\left|x^{k}\right|, z^{k}=\frac{x^{k}}{r_{k}}$, and note that along a subsequence $z^{k} \rightarrow z \in \mathbb{S}^{n-1}$ and $\tilde{u}_{k} \rightarrow u_{0}$. It follows that $\partial_{x_{1}} u_{0}(z)=N z_{n}$ and proceeding as in [IM16a] one deduces that $u_{0}(x)=a x_{1} x_{n}+c x_{n}+\tilde{b} x_{n}^{2}$ for $a \neq 0$ and $c, \tilde{b} \in \mathbb{R}$, contradicting that $u \geq 0$. This implies that in a neighborhood of the origin, there is a cone of fixed opening that can be placed below and above each free boundary point; therefore, the free boundary is Lipschitz continuous and thus $C^{1}$ by interior results [FS14, Theorem 1.3]. Since the intersection of $\Gamma$ and the origin occurs non-transversally, and

$$
\sup _{B_{2 d}^{+}\left(x^{0}\right)}|u-h| \leq \epsilon d^{2}, \quad \sup _{B_{2 d}^{+}\left(x^{0}\right)}|\nabla u-\nabla h| \leq \epsilon d,
$$

the aperture of the cones can be taken arbitrarily close to $\pi$.

Acknowledgement The author wishes to thank Donatella Danielli and Irina Mitrea for organizing the AMS Special Session "Harmonic Analysis and Partial Differential Equations" at Northeastern University. 


\section{APPENDiX}

Lemma 4.1. Let $u \in P_{r}^{+}(0, M, \Omega)$ where $\{u \neq 0\} \subset \Omega, e \in \mathbb{S}^{n-2} \cap e_{n}^{\perp}$, and suppose there exist non-negative constants $\epsilon_{0}, C_{0}$ such that $C_{0} \partial_{e} u-u \geq-\epsilon_{0}$ in $B_{r}^{+}$. Then there exists $c=c(n, \Lambda, r)>0$ such that if $\epsilon_{0} \leq c$, then $C_{0} \partial_{e} u-u \geq 0$ in $B_{\frac{r}{2}}^{+}$.

Proof. By convexity of $F$, there exist measurable uniformly elliptic coefficients $a_{i j}$ such that

$$
F\left(D^{2} u(x+h e)\right)-F\left(D^{2} u(x)\right) \geq a_{i j}\left(\partial_{i j} u(x+h e)-\partial_{i j} u(x)\right)
$$

if $x \in \Omega$ provided $h$ is small enough. Therefore,

$$
0 \geq a_{i j} \partial_{i j} \partial_{e} u \text { in } \Omega
$$

Convexity also yields

$$
a_{i j} \partial_{i j} u \geq F\left(D^{2} u(x)\right)-F(0)=1 \text { in } \Omega .
$$

Suppose now that there exists $y \in B_{\frac{r}{2}}^{+}$for which $C_{0} \partial_{e} u(y)-u(y)<0$. Let $w(x)=$ $C_{0} \partial_{e} u(x)-u(x)+\frac{|x-y|^{2}}{2 n \Lambda}$. Since $\lambda I d \leq\left(a_{i j}\right) \leq \Lambda I d$, it follows by the above that $L w \leq 0$ in $\Omega$ where $L=a_{i j} \partial_{i j}$. The maximum principle implies $\min _{\partial\left(\Omega \cap B_{r}^{+}\right)} w=$ $\min _{\Omega \cap B_{r}^{+}} w<0$. Note that $w \geq 0$ on $\partial \Omega$ and likewise on $\left\{x_{n}=0\right\}$. Therefore, the minimum occurs on $\partial B_{r}$ and thus $0>-\epsilon_{0}+\frac{1}{8 n \Lambda} r^{2}$, a contradiction if $\epsilon_{0}$ is small enough.

Remark 4.2. One may take $\epsilon_{0}=c r^{2}$, where $c>0$ depends only on the dimension and ellipticity constants of $F$.

Remark 4.3. If $u \geq 0$, then $\partial_{e_{n}} u \geq 0$ on $\left\{x_{n}=0\right\} \cap B_{r}$ and Lemma 4.1 holds therefore in this case for all $e \in \mathbb{S}^{n-1}$ such that $e \cdot e_{n} \geq 0$. 
Lemma 4.4. Let $u_{0}(x)=a x_{1} x_{n}+b x_{n}^{2}$ with $a \neq 0$ and $R \geq 1$. Then there exists $c=c(a, b)>0$ such that

$$
\inf _{D}\left|\nabla u_{0}(x)\right| \geq c
$$

where $D=\left\{x=\left(x_{1}, x^{\prime \prime}, x_{n}\right): R>|x|>R / 2,\left|x^{\prime \prime}\right| \leq \delta(R)\right\}$ for some $\delta(R)>0$.

Proof. Note $\left|\nabla u_{0}(x)\right|^{2}=a^{2} x_{n}^{2}+a^{2} x_{1}^{2}+2 a b x_{1} x_{n}+4 b^{2} x_{n}^{2}$ so that if $\left|x_{n}\right|>\frac{1}{3}$, then $\left|\nabla u_{0}(x)\right|^{2} \geq \frac{a^{2}}{9}$. If $\left|x_{n}\right| \leq \frac{1}{3}$, then for points that satisfy $\left|x^{\prime \prime}\right| \leq \sqrt{\frac{5}{72} R}$, where $x^{\prime \prime}=\left(x_{2}, x_{3}, \ldots, x_{n-1}\right)$, it follows that

$$
x_{1}^{2}>\frac{5}{72} R^{2}
$$

If $b \neq 0$, let $\epsilon^{2} \in\left(\frac{1}{a^{2}+4 b^{2}}, \frac{1}{b^{2}}\right)$. Then

$$
\begin{aligned}
\left|\nabla u_{0}(x)\right|^{2} & \geq\left(a^{2}+4 b^{2}-\frac{1}{\epsilon^{2}}\right) x_{n}^{2}+\left(a^{2}-\epsilon^{2} a^{2} b^{2}\right) x_{1}^{2} \\
& >\left(a^{2}-\epsilon^{2} a^{2} b^{2}\right)\left(\frac{5}{72} R^{2}\right) .
\end{aligned}
$$

Lemma 4.5. Let $u_{0}(x)=a x_{1} x_{n}+b x_{n}^{2}$ with $a>0$ and $R \geq 1$. Then there exists $C_{0}=C_{0}(a, b, R)>0$ such that

$$
C_{0} \partial_{x_{1}} u_{0}(x)-u_{0}(x) \geq 0
$$

in $B_{R}^{+}$

Proof. The condition is equivalent to $a x_{n}\left(C_{0}-x_{1}\right) \geq b x_{n}^{2}$. Since $x_{1} \leq R$ and $0 \leq x_{n} \leq R$, it follows that any $C_{0} \geq \frac{b}{a} R+R$ satisfies the condition.

\section{REFERENCES}

[And07] John Andersson. On the regularity of a free boundary near contact points with a fixed boundary. J. Differential Equations, 232(1):285-302, 2007.

[Caf77] Luis A. Caffarelli. The regularity of free boundaries in higher dimensions. Acta Math., 139(3-4):155-184, 1977. 
[Cha95] S. Jonathan Chapman. A mean-field model of superconducting vortices in three dimensions. SIAM J. Appl. Math., 55(5):1259-1274, 1995.

[CRS96] S. J. Chapman, J. Rubinstein, and M. Schatzman. A mean-field model of superconducting vortices. European J. Appl. Math., 7(2):97-111, 1996.

[CS02] L. Caffarelli and J. Salazar. Solutions of fully nonlinear elliptic equations with patches of zero gradient: existence, regularity and convexity of level curves. Trans. Amer. Math. Soc., 354(8):3095-3115, 2002.

[ESS98] Charles M. Elliott, Reiner Schätzle, and Barbara E. E. Stoth. Viscosity solutions of a degenerate parabolic-elliptic system arising in the mean-field theory of superconductivity. Arch. Ration. Mech. Anal., 145(2):99-127, 1998.

[FS14] Alessio Figalli and Henrik Shahgholian. A general class of free boundary problems for fully nonlinear elliptic equations. Arch. Ration. Mech. Anal., 213(1):269-286, 2014.

[IM16a] Emanuel Indrei and Andreas Minne. Nontransversal intersection of free and fixed boundaries for fully nonlinear elliptic operators in two dimensions. Anal. PDE, $9(2): 487-502,2016$.

[IM16b] Emanuel Indrei and Andreas Minne. Regularity of solutions to fully nonlinear elliptic and parabolic free boundary problems. Ann. Inst. H. Poincaré Anal. Non Linéaire, 33(5):1259-1277, 2016.

[Ind18] Emanuel Indrei. Non-transversal intersection of the free and fixed boundary in the mean-field theory of superconductivity. arXiv:1810.08724, 2018.

[Ind19] Emanuel Indrei. Boundary regularity and non-transversal intersection for the fully nonlinear obstacle problem. Comm. Pure Appl. Math., doi.org/10.1002/cpa.21814, 2019 .

[KN77] D. Kinderlehrer and L. Nirenberg. Regularity in free boundary problems. Ann. Scuola Norm. Sup. Pisa Cl. Sci. (4), 4(2):373-391, 1977.

[MM04] Norayr Matevosyan and Peter A. Markowich. Behavior of the free boundary near contact points with the fixed boundary for nonlinear elliptic equations. Monatsh. Math., 142(1-2):17-25, 2004.

[PSU12] Arshak Petrosyan, Henrik Shahgholian, and Nina Uraltseva. Regularity of free boundaries in obstacle-type problems, volume 136 of Graduate Studies in Mathematics. American Mathematical Society, Providence, RI, 2012. 
[Saf94] M.V. Safonov. On the boundary value problems for fully nonlinear elliptic equations of second order. Mathematics Research Report No. MRR 049-94, Canberra: The Australian National University, 1994.

[Sch77] David G. Schaeffer. Some examples of singularities in a free boundary. Ann. Scuola Norm. Sup. Pisa Cl. Sci. (4), 4(1):133-144, 1977.

[SU03] Henrik Shahgholian and Nina Uraltseva. Regularity properties of a free boundary near contact points with the fixed boundary. Duke Math. J., 116(1):1-34, 2003.

[Ura96] N. N. Uraltseva. $C^{1}$ regularity of the boundary of a noncoincident set in a problem with an obstacle. Algebra i Analiz, 8(2):205-221, 1996.

\author{
EMANUEL INDREI \\ Department of Mathematics \\ Purdue University \\ West LAFAYETTE, IN 47907, USA \\ EMAIL: eindrei@purdue.edu
}

